\title{
Numerical Study of a Novel Variable Diameter Cavitator Structure
}

\author{
Zeyang Xie ${ }^{1}$, Min Xiang ${ }^{1}$, Bo Liu ${ }^{1}$, Weihua Zhang ${ }^{1}$ \\ ${ }^{1}$ College of Aerospace Science and Technology, National University of Defense Technology \\ No.109 Deya Road, Changsha, Hunan, 410073, P.R. China \\ xiezeyang@ @udt.edu.cn; xiangmin333@ hotmail.com; \\ lb123456@outlook.com; zhangweihua@nudt.edu.cn
}

\begin{abstract}
Supercavitation is a perspective method for drag reduction. Cavitator is one of the most crucial parts of a supercavitation vehicle and cause $70 \% \sim 80 \%$ of total drag. So, the design of cavitator remains a key issue. The requirements of diameter differ at various speed and it is difficult for cavitators with fixed structure to be adapted to complex operating conditions. In this paper, a novel variable diameter cavitator is designed to solve this problem. Based on ANSYS Fluent dynamic mesh techniques and UDF (user-defined function), three-dimensional CFD (computational fluid dynamics) simulations are performed to investigate the variation rules. According to a series of results from numerical simulation, hydrodynamic characteristic of variable diameter cavitator is obtained. The corresponding cavitator structural configuration are designed for realizing the function of changing the diameter. Results show that there is a nonlinear hysteresis property of cavity deforming affected by variable diameter cavitator.
\end{abstract}

Keywords: cavitator, Numerical simulation, Supercavitation, Dynamic mesh

\section{Introduction}

Supercavitation is a prevalent area of importance and research interest. Gas has a lower viscosity than the liquid and once the moving object is enveloped by a large and continuous cavity, friction drag will be reduced a lot. Generally, there are two approaches to generate a stable supercavity: one is to achieve high speed near the nose of the body, which is termed vaporous cavitation. And the other way is to entrain gas into a cavity, called artificial cavitation or ventilated cavitation. The latter one possesses great advantage in active control for underwater vehicles.

According to the relative motion between the object and the liquid, cavitation state can be divided as noncavitation, critical cavitation, partial cavitation and supercavitation. Supercavitation is the stage when the cavity dimensions considerably exceed the body dimensions and then the friction drag can be greatly reduced.

Cavitator is one of the most crucial parts of a supercavitation vehicle, which generates a cavity of sufficient size to envelop the body to minimize drag and sustain the form of a supercavity. Conditions for cavitation are created by inducing depressurization by vortex flow. This generation ability determines the navigation performance and dynamic characteristics. So, the design of a cavitator does make sense and play a key role in the design of an underwater vehicle.

At present, there are a series of systematic studies on traditional cavitators but few prior studies on variable structure cavitators. Logvinovich [1] conducted a large number of theoretical and experimental studies on the disk cavitator, and constructed mathematical calculation formulas for cavitation number, diameter of the disk cavitator, and cavity dimensions. The institute of hydrodynamics of the national academy of sciences of Ukraine proposed several cavitators with potential utility, such as conical and polygonal cavitators Stinebring D R et al. [2] put forward the concept of variable cavitator, which consists of a smooth low-drag center body that can move within a cylindrical housing. Cavity size and drag can be controlled by extending or retracting the center body. The main aim is to change in location of the cavity leading edge. In conclusion, the concept of variable cavitator proved to be feasible in this experiment.

Vlasenko Y D et al. [3] used a variable drag cavitator model to study on parameters control of ventilated cavitator. Huang et al. [4] solved the problem of numerical simulation on supercavity induced by disk-cavitator with attack angle. Fard M B et al. [5] studied on unsteady turbulent cavitating flows around a circular disk and a cone cavitator. Kwack et al. [6] used a diffuse interface model to compute axisymmetric supercavitating flows around various cavitators.

Therefore, in this study, variable disk cavitator is designed to adapt to various speed. It's an efficient method for active control and drag reduction. While an underwater vehicle cruising at extreme speed, a larger cavitator, which is designed to meet low-speed mission requirements, will generate unnecessary energy losses. Once the diameter of cavitator varies as 
navigation speed and background pressure, it will be more efficient. At the same time, it also offsets the impact of any such as change in vehicle trust, vehicle depth and marine environmental disturbance.

Typical cavitator configuration includes disk shape and conical shape. Limited by its capability of generating cavity of enough size, conical cavitator is not suitable enough to a variable diameter structure. For the purpose of diameter being adjustable, its scope for adjustment is even smaller. As a result, disk cavitator is chosen in this paper.

Supercavitating flows it considerably depends on the cavitation number and the Froude number.

$$
\begin{gathered}
\sigma_{c}=\frac{2\left(p_{\infty}-p_{c}\right)}{\rho_{\infty} U_{\infty}^{2}} \\
F r=\frac{U_{\infty}}{\sqrt{g D_{n}}} \\
C_{q}=\frac{Q_{D}}{\rho U_{\infty} D_{n}^{2}}
\end{gathered}
$$

Where $p_{\infty}$ is the hydrostatic pressure, $p_{c}$ is the pressure in the cavity, $\rho$ is water density, $U_{\infty}$ is the mainstream velocity, $D_{n}$ is diameter of the cavitator and $Q_{D}$ is mass flow rate of noncondensable gas.

In this paper, two phase flow problem is studied to investigate the ventilated supercavity around underwater vehicle. Firstly, the structural configuration arrangement of variable diameter cavitator and dynamic mesh setup are shown. Secondly, the flow characteristic and cavity closures are investigated to understand the influence of this novel structure.

\section{Governing equations}

\subsection{Basic governing equations}

Cavitating flows satisfy the conservation of mass, momentum and phase volume fraction.

The continuum equation of mixed media is

$$
\frac{\partial \rho_{m}}{\partial t}+\frac{\partial\left(\rho_{m} u_{i}\right)}{\partial x_{i}}=0
$$

Where, $u$ is the velocity of the mixed flow, and $i$ is the Cartesian direction, $\rho_{m}$ represents the density of the mixed flow. Ignoring the influence of gravity and surface tension, the momentum equation of the mixed medium is

$$
\frac{\partial\left(\rho_{m} u_{i}\right)}{\partial t}+\frac{\partial\left(\rho_{m} u_{i} u_{j}\right)}{\partial x_{i}}=-\frac{\partial p}{\partial x_{i}}+\frac{\partial}{\partial x_{j}}\left[\mu_{m}\left(\frac{\partial u_{i}}{\partial x_{j}}+\frac{\partial u_{j}}{\partial x_{i}}\right)\right]+f
$$

Where, $\mathrm{p}$ is the pressure of the mixed flow, and the subscript $\mathrm{i}$ and $\mathrm{j}$ are the Cartesian directions. $\mathrm{f}$ is the momentum source terms due to surface tension and gravity:

$$
f=F_{\sigma}+\rho_{m} g
$$

\subsection{VOF method}

Volume-of-Fluid (VOF) method is used to deal with surface tracking problems for immiscible fluids.

Volume fraction $\alpha$ defined in two-phase system are:

$$
\begin{gathered}
\alpha_{l}=\frac{V_{l}}{V} \\
\alpha_{g}=\frac{V_{g}}{V}
\end{gathered}
$$

Where $\alpha_{l}$ and $\alpha_{g} \quad V_{l}$ and $V_{g}$ represent the volume occupied by liquid and gas in the control unit. $V$ is the total of volume of control unit. A step function is defined as:

$$
\alpha_{l}=\left\{\begin{array}{cc}
1 & \text { control unit is filled wih liquid } \\
0<\alpha<1 & \text { mixture } \\
0 & \text { control unit is filled wih gas }
\end{array}\right.
$$


Additionally, density of mixture $\rho_{m}$ and viscosity coefficient $\mu_{m}$ is weighted by volume fraction

$$
\left\{\begin{array}{l}
\rho_{m}=\rho_{g} \alpha_{g}+\rho_{l} \alpha_{l} \\
\mu_{m}=\mu_{g} \alpha_{g}+\mu_{l} \alpha_{l}
\end{array}\right.
$$

The volume fraction equations can be expressed as:

$$
\frac{\partial \alpha_{l}}{\partial t}+\frac{\partial\left(\alpha_{l} u_{i}\right)}{\partial x_{i}}+\frac{\partial}{\partial x_{i}} \alpha_{l}\left(1-\alpha_{l}\right) u_{C i}=0
$$

\subsection{Turbulence model}

The cavitation flow field, especially the closed cavity region, is filled with steam, liquid drops and vortices. The influence of turbulence must be considered in the numerical simulation. In this study, the standard k-epsilon turbulence model is adopted to solve the cavitation flow field. The transport equations for the turbulent kinetic energy $\mathrm{k}$ and dissipation rate $\varepsilon$ are presented in Eq. (8) and Eq. (9)

$$
\begin{gathered}
\frac{\partial\left(\rho_{m} k\right)}{\partial t}+\frac{\partial\left(\rho_{m} u_{i} k\right)}{\partial x_{i}}=P_{k}-\rho_{m} \varepsilon+\frac{\partial}{\partial x_{i}}\left[\left(\mu_{m}+\frac{\mu_{t}}{\sigma_{k}}\right) \frac{\partial k}{\partial x_{i}}\right] \\
\frac{\partial\left(\rho_{m} \varepsilon\right)}{\partial t}+\frac{\partial\left(\rho_{m} u_{i} \varepsilon\right)}{\partial x_{i}}=C_{1 \varepsilon} \frac{\varepsilon}{k} P_{k}-C_{2 \varepsilon} \rho_{m} \frac{\varepsilon^{2}}{k}+\frac{\partial}{\partial x_{j}}\left[\left(\mu_{m}+\frac{\mu_{t}}{\sigma_{\varepsilon}}\right) \frac{\partial \varepsilon}{\partial x_{j}}\right]
\end{gathered}
$$

Where, $\mu_{t}$ is turbulent viscosity, and $P_{k}$ is the turbulence production term due to viscous forces, while $C_{1 \varepsilon}, C_{2 \varepsilon}, \sigma_{\varepsilon}$ $\sigma_{k}$ are the model empirical constants. The turbulent dynamic viscosity is defined as

$$
\mu_{t}=\rho_{m} C_{\mu} \frac{k^{2}}{\varepsilon}
$$

Where $\mathrm{C} \mu$ is not a constant by a complicated function.

\section{Computation domain and grid}

The computation domain is $1 / 2$ cylindrical area with a diameter of 15 times the vehicle diameter. And a length of 20 vehicle body length is adopted. The entrance of the calculation field is about 5 times the vehicle length from the head of the model, and the downstream exit distance is about 15 times the vehicle length from the head of the model.

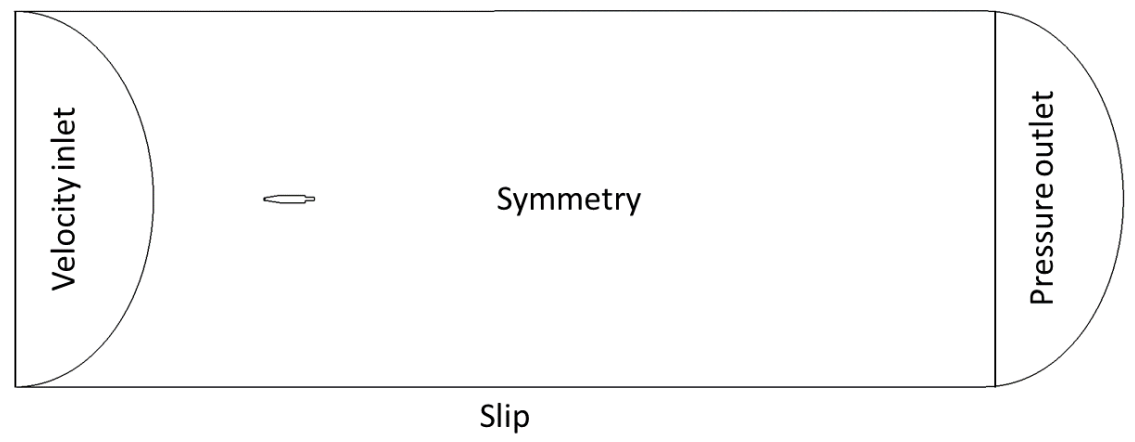

Fig. 1: Computation domain and boundary conditions.

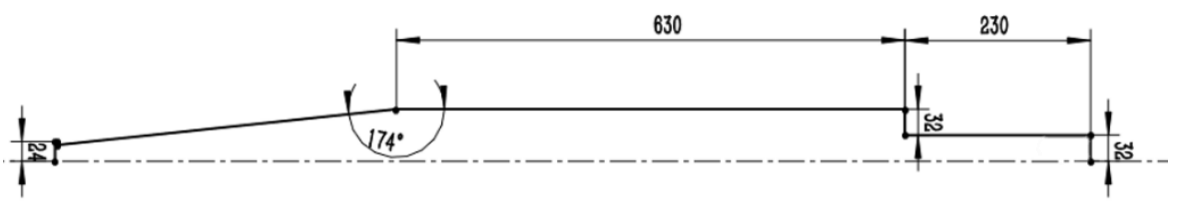

Fig. 2: The model size of vehicle ( $\mathrm{mm})$. 


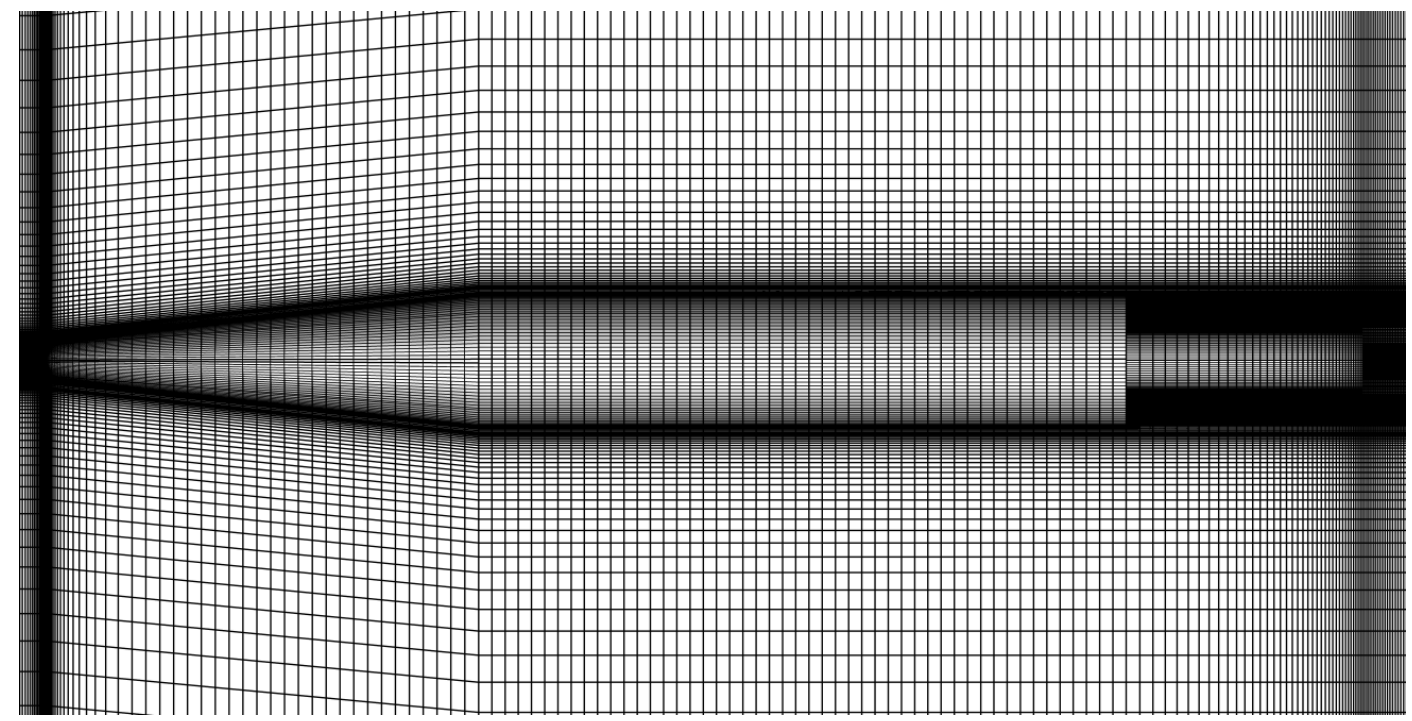

Fig. 3: Grid distribution.

In order to and investigate the hydrodynamic characteristic of this novel structure and simulate it properly, ANSYS Fluent dynamic mesh technique and UDF (user-defined function) is used in defining the parameters of this dynamic model and update the grid after edge of outer cavitator diameter movement.

The schematic diagram of grid motion is shown in Fig. 4 and Fig. 5. Methods of layering enable layers to add or remove. Ideal height is set as the cell height adjacent to moving boundary. The grid motion is defined by UDF, which specifies the radial motion of grid points in specific zones.

Specifically, we dispersed the continuous process of diameter variation into 10 steps and that means mesh nodes moves radially at every step and stays for 0.5 seconds to avoid inertia affecting the subsequent results. The average velocity of grid motion is set as $2 \mathrm{~mm} / \mathrm{s}$ and remains the same, which results in diameter increase at the speed of $4 \mathrm{~mm} / \mathrm{s}$ in total.

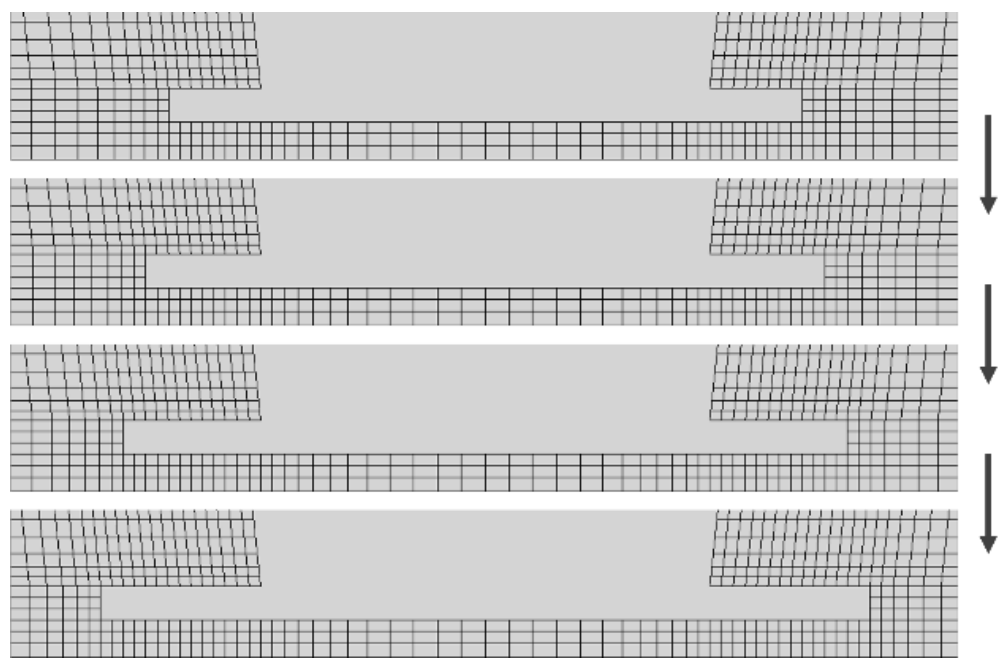

Fig. 4: An example of grid motion in horizontal cut plane around disk cavitator. 


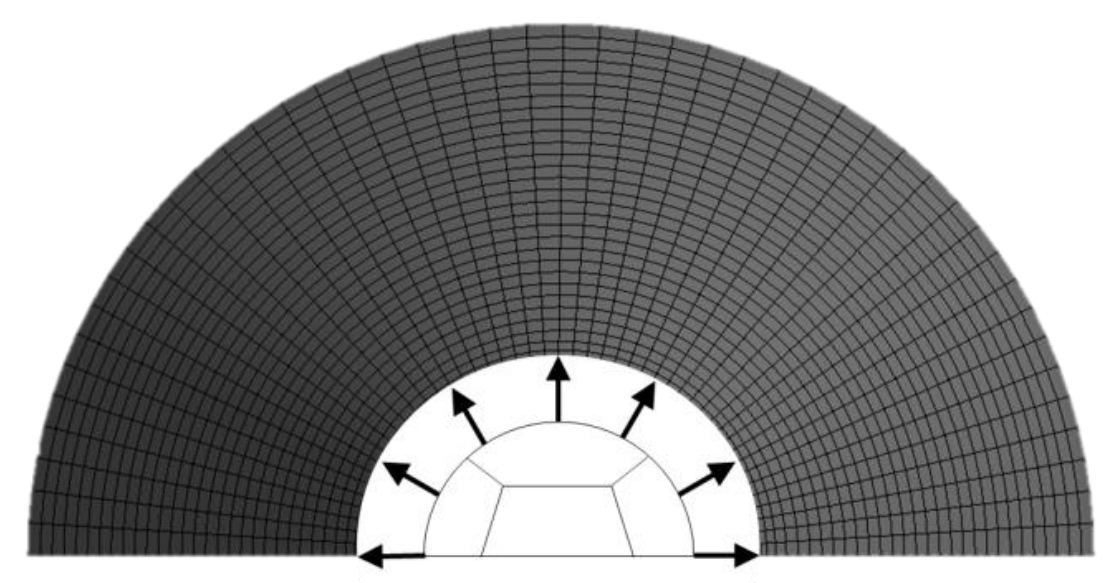

Fig. 5: An example of grid motion in vertical cut plane around disk cavitator.

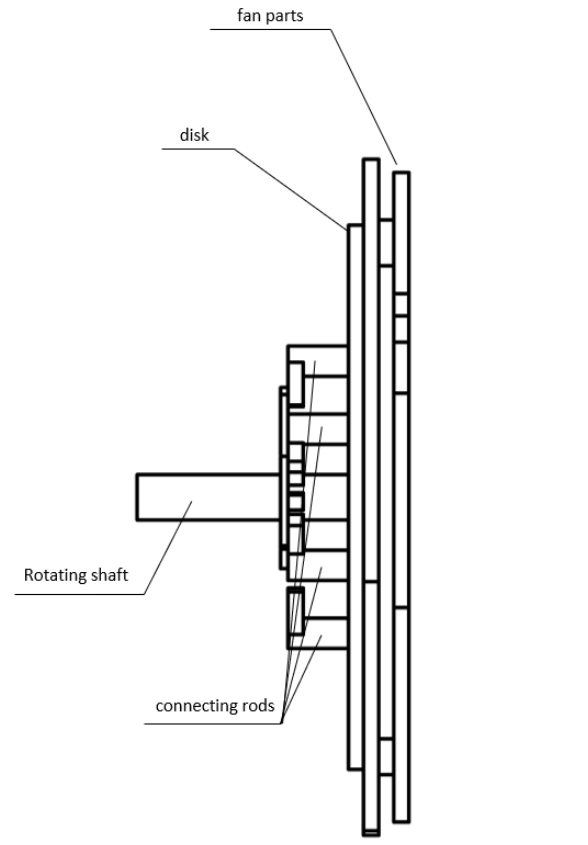

Fig. 6: Schemes of mechanical continuously variable diameter cavitation structure (side view).

A scheme of mechanical continuously variable diameter cavitatior structure is depicted in Fig.6. The main components include a rotating shaft, connecting rods and a driving shaft. Central rotating shaft is driven by a servo motor, which is powered by a battery pack. At the same time, the fan parts are driven to rotate, and their rims move inward (or outward) synchronously, resulting in the change of outline dimensions. By adjusting the shape of cavitator at different speed, the size of the cavity can be actively controlled so that the supercavitation will finally envelope the vehicle properly and reduce the resistance on the surface of the vehicle. In this study, the designed diameter range is $48 \mathrm{~mm}$ to $68 \mathrm{~mm}$, which is approximately $1 / 3$ to $1 / 2$ of cylinder diameter, respectively. 


\section{Results and discussion}

Former experiments show that the rear portion of the cavity is unsteady because of the complicated interaction between steam, noncondensable gas, liquid drops and vortices. It expands and shrinks periodically, and its dimension is largely predetermined by its closure modes.

The following results of dynamic mesh simulation show there is a significant link between the history of ventilated cavity and cavity closure modes at constant mass flow rate. The hydrodynamic characteristics of the ventilated cavitating flow are related to Froude number based on cavitator diameter $\mathrm{Fr}$ and cavitation number $\sigma_{c}$.

Table 1: Working conditions for ventilated cavitating flow ( $\left.U_{\infty}=60 \mathrm{~m} / \mathrm{s}\right)$.

\begin{tabular}{ccccccc}
\hline Time & $0.00 \mathrm{~s}$ & $1.00 \mathrm{~s}$ & $2.00 \mathrm{~s}$ & $3.00 \mathrm{~s}$ & $4.00 \mathrm{~s}$ & $5.00 \mathrm{~s}$ \\
\hline Fr & 87.48 & 84.04 & 80.99 & 78.25 & 75.76 & 73.50 \\
$\sigma_{c}$ & 0.020 & 0.028 & 0.036 & 0.037 & 0.038 & 0.047 \\
\hline Time & $10.00 \mathrm{~s}$ & $9.00 \mathrm{~s}$ & $8.00 \mathrm{~s}$ & $7.00 \mathrm{~s}$ & $6.00 \mathrm{~s}$ & $5.00 \mathrm{~s}$ \\
\hline $\mathrm{Fr}$ & 87.48 & 84.04 & 80.99 & 78.25 & 75.76 & 73.50 \\
$\sigma_{c}$ & 0.047 & 0.042 & 0.040 & 0.035 & 0.015 & 0.010 \\
\hline
\end{tabular}




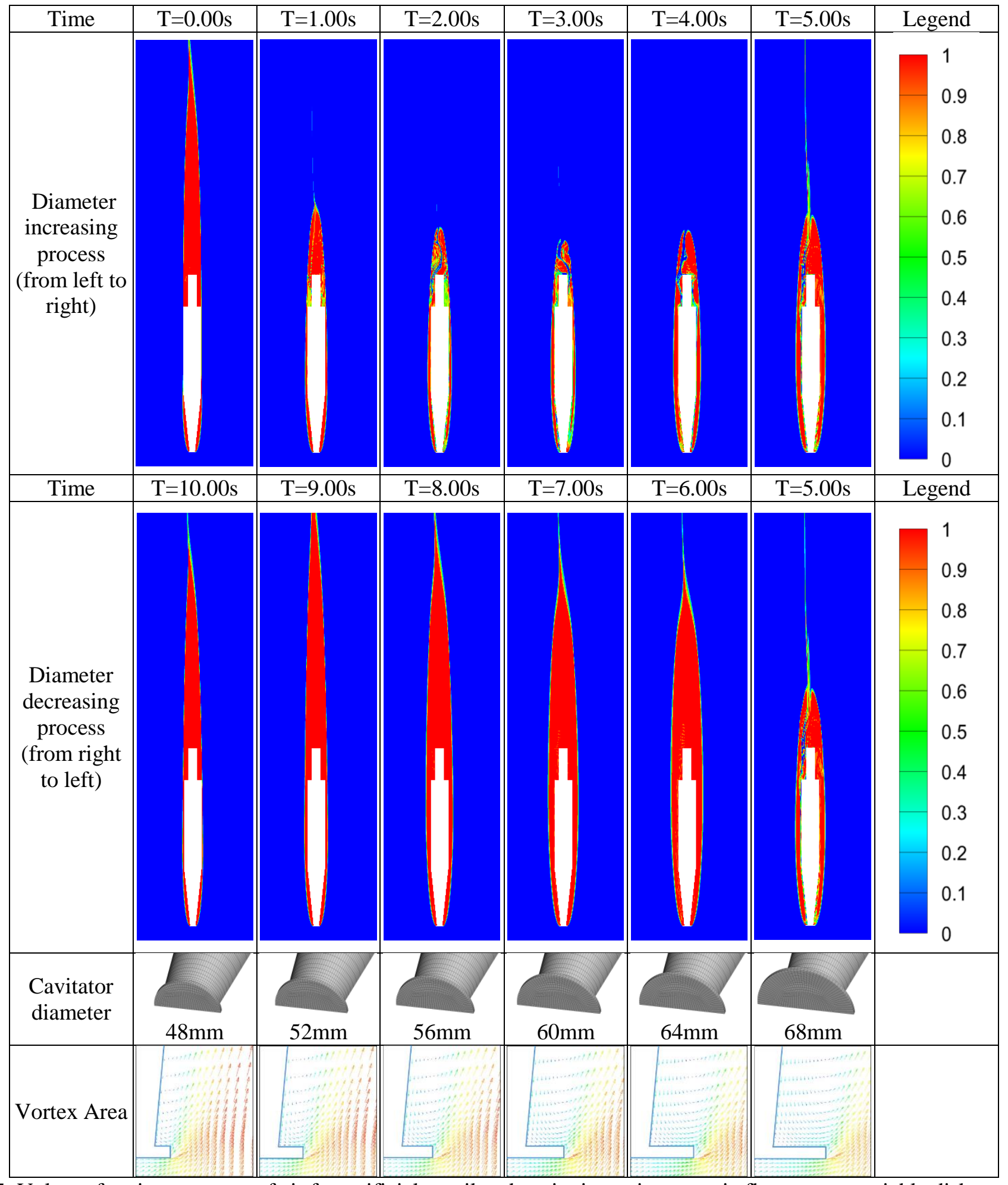

Fig. 7: Volume fraction contours of air for artificial ventilated cavitating axisymmetric flow over a variable disk cavitator configuration $\left(U_{\infty}=60 \mathrm{~m} / \mathrm{s}\right)$. 
Fig. 7 demonstrated the transition between the two closure types, i.e. the re-entrant jet (RJ) and the twin vortex (TV) and it is deeply influenced by the change process over cavitator. The first is characterized by an reversed water jet penetrating the cavity, which is known as the RJ closure. And at large ventilation rate and small Froude number, the ventilation gas sheds through two stationary vortex tubes, known as TV closure.

The ventilation port is located at the back end of the cavitator and its boundary condition is specified as mass flow inlet, which is specified as $0.025 \mathrm{~kg} / \mathrm{s}$ and keeps the same level among all the conditions.

A series of experiments were carried out by G.V. Logvinovich [7] to verify the principle of independence of cavity section expansion. According to this principle, whether it's increasing or decreasing, cavity section around cavitator varies at every moment and extend to downstream. Specifically, every cavity section expands independently, and it is hardly dependent on the former motion or later motion. When fluid begins flowing through the cavitator, it obtains kinetic energy from cavitator, which decays in overcoming resistance in the flow. Therefore, the whole process seems to be symmetrical.

However, under the same ventilation rate and cavitator diameter $D_{n}$, cavity dimensions and closure types yield some difference. In the beginning, the initial field is set as steady result of ventilated cavitating axisymmetric flow at a diameter of $48 \mathrm{~mm}$. The cavity attached on the vehicle started from partial cavity and a base cavity is formed near the rear portion of the vehicle where gas exits from the vortex tubes due to buoyancy. As expected, cavity diameter increases gradually and envelope the whole vehicle. But surprisingly, the closure mode will transfer from TV to RJ soon. It is difficult to maintain a stable cavity topology as the cavity collapses and its dimension shrinks rapidly when cavity boundary expands. The following 5 seconds witnesses the pulse of cavity closure around 1.2L.

As for RJ closure, Kelvin-Helmholtz vortices are rolled up when water penetrates the liquid-gas interface and causes instability near shear interface. It is estimated that strong disturbance is due to diameter expansion in the increasing process, which may lead to the air mass is leaking out of the cavity continuously.

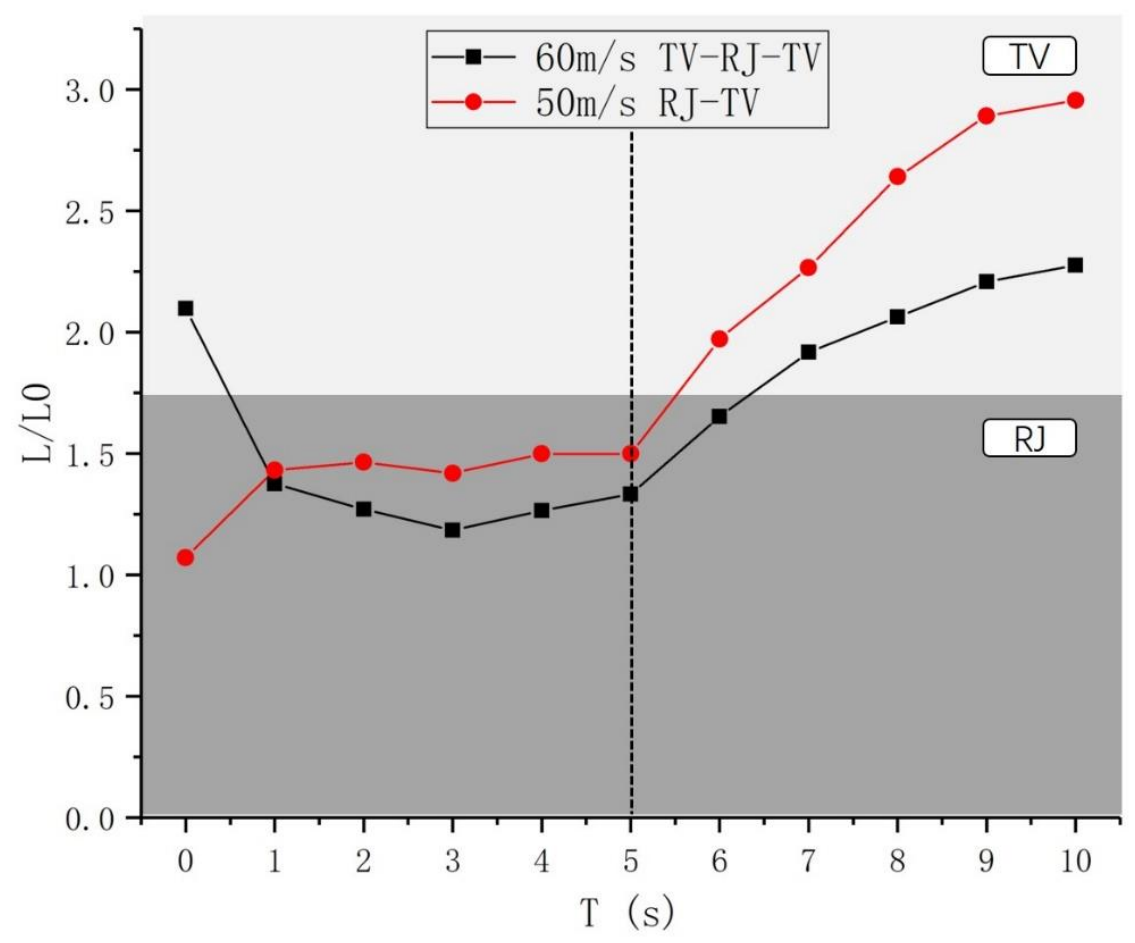

Fig. 8: Dimensionless cavity length against time. 
Fig. 8 demonstrates the graph of the dimensionless cavity length against time, where $L_{0}$ represents the total length of the vehicle and L represents the cavity length. To the left of the dotted line is the increasing process, and the right side is the opposite. Numerical simulation of two groups at different navigational speed shows that the variable diameter structure keeps good consistency to each working condition under the same process.

In a similar way, the scheme of variable diameter cavitator makes sense while maneuvering. The criteria of maneuverability for supercavitation vehicles is various, but one thing in common it that the larger the wet area is, the worse maneuverability is. As the vehicle depth increases, cavity dimensions plunge and then wet surface expands, which results in variation of hydrodynamic characteristics. Cavity profile is enlarged to proper by ways of adjusting the diameter. In addition, a better matching should be sought between control surfaces and cavitator.

\section{Conclusion}

The following conclusions are drawn through transient dynamic mesh numerical simulation.

Variable diameter cavitator structure is feasible and adapts to a variety of working conditions. The development of new variable structure cavitator is a new idea for developing new underwater vehicles. Its superiority over traditional cavitator lies in flexibility and economics.

There is a hysteresis characteristic of cavity deforming affected by variable diameter cavitator through transient dynamic mesh simulation. Results show the significant link between the history of ventilated cavity and cavity closure modes. Diameter variation has a great impact on cavity stability and there may a transition from TV to RJ closure in increasing process while there may be a transition from RJ to TV closure in decreasing process.

\section{References}

[1] G. V. Logvinovich and V. N. Buyvol, "Hydrodynamics of cavitating flows with perturbations. A translation of Sovremen. Probl. Gidromeh. i Gidroteh. 1977," vol. 110, no. 9, pp. 559-564, 2003.

[2] D. R. Stinebring, R. B. Cook, J. E. Dzielski, and R. F. Kunz, "High-speed supercavitating vehicles," in AIAA Guidance, Navigation, and Control Conference 2006, 2006, pp. 3092-3099.

[3] Y. D. Vlasenko, "Control of cavity parameters at supercavitating flow," High Speed Body Motion in Water, p. 18, 1998.

[4] H.-l. HUANG, C. WANG, W.-h. HUANG, C.-b. ZHAO, and F. ZHOU, "Numerical Simulation on Natural Supercavity Based on Disk-Cavitator with Variable Attack Angle [J]," Transactions of Beijing Institute of Technology, vol. 2, pp. 100-103, 2008.

[5] M. B. Fard and A. Nikseresht, "Numerical simulation of unsteady 3D cavitating flows over axisymmetric cavitators," Scientia Iranica, vol. 19, no. 5, pp. 1258-1264, 2012.

[6] Y. K. Kwack and S. H. Ko, "Numerical analysis for supercavitating flows around axisymmetric cavitators," International Journal of Naval Architecture and Ocean Engineering, vol. 5, no. 3, pp. 325-332, 2013, doi: 10.2478/ijnaoe-2013-0136.

[7] G. V. Logvinovich, "Hydrodynamics of Flows with Free Boundaries," 1969.

[8] Y. Savchenko, "Supercavitation-problems and perspectives," http://resolver. caltech. edu/cav2001: lecture. 003, 2001. 